\title{
Tracking the critical point of strongly interacting matter through proton intermittency analysis in NA61/SHINE
}

\author{
Nikolaos Davis ${ }^{a, 1, *}$ \\ ${ }^{a}$ Institute of Nuclear Physics PAN, \\ ul. Radzikowskiego 152, Kraków, Poland \\ E-mail: nikolaos.davis@ifj.edu.pl
}

The search for experimental signatures of the critical point (CP) of strongly interacting matter is one of the main objectives of the NA61/SHINE experiment at CERN SPS. In the course of the experiment, a beam momentum $(13 \mathrm{~A}-150 \mathrm{~A} \mathrm{GeV} / \mathrm{c})$ and system size $(\mathrm{p}+\mathrm{p}, \mathrm{p}+\mathrm{Pb}, \mathrm{Be}+\mathrm{Be}, \mathrm{Ar}+\mathrm{Sc}$, $\mathrm{Xe}+\mathrm{La}, \mathrm{Pb}+\mathrm{Pb})$ scan is performed. Local proton density fluctuations in transverse momentum space represent an order parameter of the chiral phase transition and are expected to scale according to a universal power-law in the vicinity of the CP. They can be probed through an intermittency analysis of the proton second scaled factorial moments (SSFMs) in transverse momentum space. Previous such analyses [1] revealed power-law behavior in NA49 Si+Si collisions at 158A GeV/c, the fitted power-law exponent being consistent with the theoretically expected critical value, within errors. Probes of NA61/SHINE systems at the maximum SPS energy revealed no intermittency effect in $\mathrm{Be}+\mathrm{Be}$, whereas $\mathrm{Ar}+\mathrm{Sc}$ analysis is inconclusive due to large uncertainties. The analysis has recently been extended to $\mathrm{Pb}+\mathrm{Pb}$ collision data at lower energies.

We present a summary of the current status of NA61/SHINE intermittency analysis, and review novel techniques developed and employed to subtract non-critical background and estimate statistical and systematic uncertainties. Additionally, we use Monte Carlo simulations to assess the statistical significance of the observed intermittency effect.

\footnotetext{
*** The European Physical Society Conference on High Energy Physics (EPS-HEP2021), ***

*** 26-30 July $2021 * * *$

*** Online conference, jointly organized by Universität Hamburg and the research center DESY ***
}

\footnotetext{
${ }^{1}$ For the NA61/SHINE collaboration.

* Speaker
} 


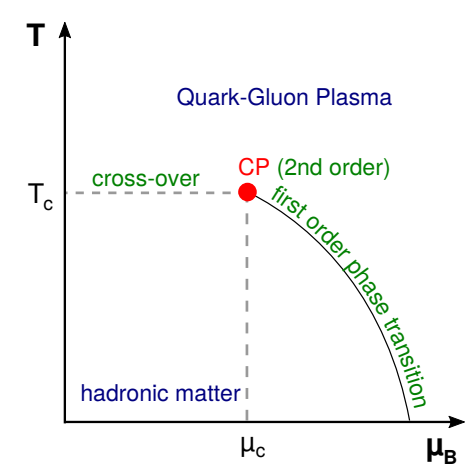

Figure 1: Hypothetical sketch of the phase diagram of strongly interacting matter with critical point, drawn as a function of baryochemical potential $\mu_{B}$ and temperature $T$.

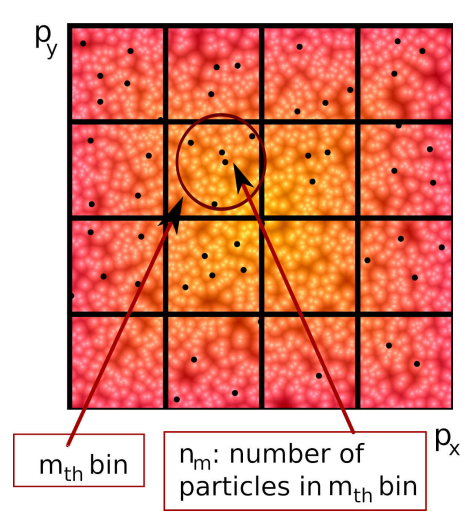

Figure 2: Counting proton pairs in a transverse momentum space partitioning of $M \times M$ equal size bins. [Image by I. Sputowska]

\section{Introduction}

NA61/SHINE is a fixed target particle and high-energy nuclear physics experiment at CERN SPS [2], colliding a variety of beams on hydrogen and nuclear targets. One of the physics goals of its strong interactions programme is to search for experimental signatures of the critical point (CP) of strongly interacting matter. To this purpose, a scan is performed of the phase diagram of strongly interacting matter, varying system size $(\mathrm{Be}+\mathrm{Be}, \mathrm{Ar}+\mathrm{Sc}, \mathrm{Xe}+\mathrm{La}, \mathrm{Pb}+\mathrm{Pb})$ and collision energy $(13 \mathrm{~A}-150 \mathrm{~A} \mathrm{GeV} / c)$, thus probing different freeze-out conditions in temperature $T$ and baryochemical potential $\mu_{B}$ (Fig.1).

A characteristic feature of a second order phase transition (expected to occur at the $\mathrm{CP}$ ) is the divergence of the correlation length, leading to a scale-invariant system effectively described by a universality class. Of particular interest are local fluctuations of the order parameter of the QCD chiral phase transition, the chiral condensate $\sigma(\mathbf{x})=\langle\bar{q}(\mathbf{x}) q(\mathbf{x})\rangle$. At finite baryochemical potential, the critical fluctuations of the chiral condensate are transferred to the net-baryon density [3]. For a critical system, we expect proton density fluctuations to be self-similar [4], obeying power-laws with critical exponents determined by the 3D Ising universality class [5-7]. Such fluctuations correspond to a power-law scaling of the proton density-density correlation function, which can be detected in transverse momentum space within the framework of an intermittency analysis [7, 8] of proton scaled factorial moments (SFMs). A detailed analysis can be found in Ref. [1], where we study various heavy nuclei collision datasets recorded in the NA49 experiment at maximum energy (158A GeV/c, $\sqrt{s_{N N}} \approx 17 \mathrm{GeV}$ ) of the SPS (CERN).

\section{Methodology}

Intermittency analysis examines how the Second Scaled Factorial Moments (SSFM) $F_{2}(M)$ of proton transverse momenta scale with the number of $2 \mathrm{D}$ bins $M^{2}$ at mid-rapidity (Fig.2):

$$
F_{2}(M) \equiv\left\langle\frac{1}{M^{2}} \sum_{i=1}^{M^{2}} n_{i}\left(n_{i}-1\right)\right\rangle /\left\langle\frac{1}{M^{2}} \sum_{i=1}^{M^{2}} n_{i}\right\rangle^{2}
$$

where $n_{i}$ is the number of protons in the $i$-th bin, and $\langle\ldots\rangle$ denotes average over events. 
For a pure critical system, $F_{2}(M)$ is predicted to follow a power-law [7]:

$$
F_{2}(M) \sim M^{2 \cdot \phi_{2, c r}} \quad, \quad \phi_{2, c r}^{(p)}=5 / 6
$$

For a noisy system, mixed event moments must be subtracted from the data moments in order to recover the critical component [1]. Thus, we define the correlator $\Delta F_{2}(M)$ :

$$
\Delta F_{2}(M)=F_{2}^{(d)}(M)-F_{2}^{(m)}(M)
$$

SSFMs statistical errors are estimated by the bootstrap method [9], whereby the original set of events is resampled with replacement.

\section{Results}

Intermittency analysis of peripheral $\mathrm{Ar}+\mathrm{Sc}$ collisions at $150 \mathrm{~A} \mathrm{GeV/c}$ (Fig.3a) reveals a nontrivial scaling effect; however, large uncertainties in $F_{2}(M)$ and $M$-bin error correlations [10] prevent an unbiased estimation of $\phi_{2}$ confidence intervals. By comparing the $\mathrm{Ar}+\mathrm{Sc} F_{2}(M)$ effect to random Monte Carlo background (Fig.3b), we obtain an estimate of its statistical significance: $\sim 1-2 \sigma$ from a zero effect, approximately.

Alternatively, one can use independent bins, where a different subset of events is used to calculate $F_{2}(M)$ for each $M$-bin. This eliminates bin correlations at the cost of very significantly reducing per bin event statistics. Independent bin analysis using cumulative variables [11] shows no intermittency effect in $\mathrm{Ar}+\mathrm{Sc}$ at $150 \mathrm{~A} \mathrm{GeV} / c$ (Fig.3c), as well as in $\mathrm{Pb}+\mathrm{Pb}$ at $30 \mathrm{~A} \mathrm{GeV} / c$ (Fig.3d).

\section{Conclusions}

Proton intermittency analysis is a promising tool for detecting the critical point of strongly interacting matter. However, it poses certain challenges, requiring large event statistics as well as careful handling of bin correlations.

The scan performed by NA61/SHINE in collision energy and system size currently observes no intermittency effect in $\mathrm{Pb}+\mathrm{Pb}$ collisions at $30 \mathrm{~A} \mathrm{GeV} / c$, and $\mathrm{Ar}+\mathrm{Sc}$ collisions at $150 \mathrm{~A} \mathrm{GeV} / c$ when using independent bin analysis. Evidence is still inconclusive for $\mathrm{Ar}+\mathrm{Sc}$ collisions at $150 \mathrm{~A} \mathrm{GeV} / \mathrm{c}$ analyzed through the conventional (correlated bin) method. Proper $\phi_{2}$ confidence interval estimation is still pending. Statistical and systematic uncertainties are being investigated via Monte Carlo models \& the statistical bootstrap method.

Acknowledgments: This work was supported by the National Science Centre, Poland (grant no. 2014/14/E/ST2/00018).

\section{References}

[1] NA49 collaboration, Critical fluctuations of the proton density in A+A collisions at 158A GeV, Eur. Phys. J. C 75 (2015) 587 [1208 . 5292].

[2] N. Abgrall, O. Andreeva, A. Aduszkiewicz, Y. Ali, T. Anticic, N. Antoniou et al., NA61/SHINE facility at the CERN SPS: beams and detector system, Journal of Instrumentation 9 (2014) P06005.

[3] Y. Hatta and M.A. Stephanov, Proton number fluctuation as a signal of the QCD critical endpoint, Phys. Rev. Lett. 91 (2003) 102003 [hep-ph/0302002].

[4] T. Vicsek, Fractal Growth Phenomena, ISBN: 9789971508302, World Scientific (1989). 

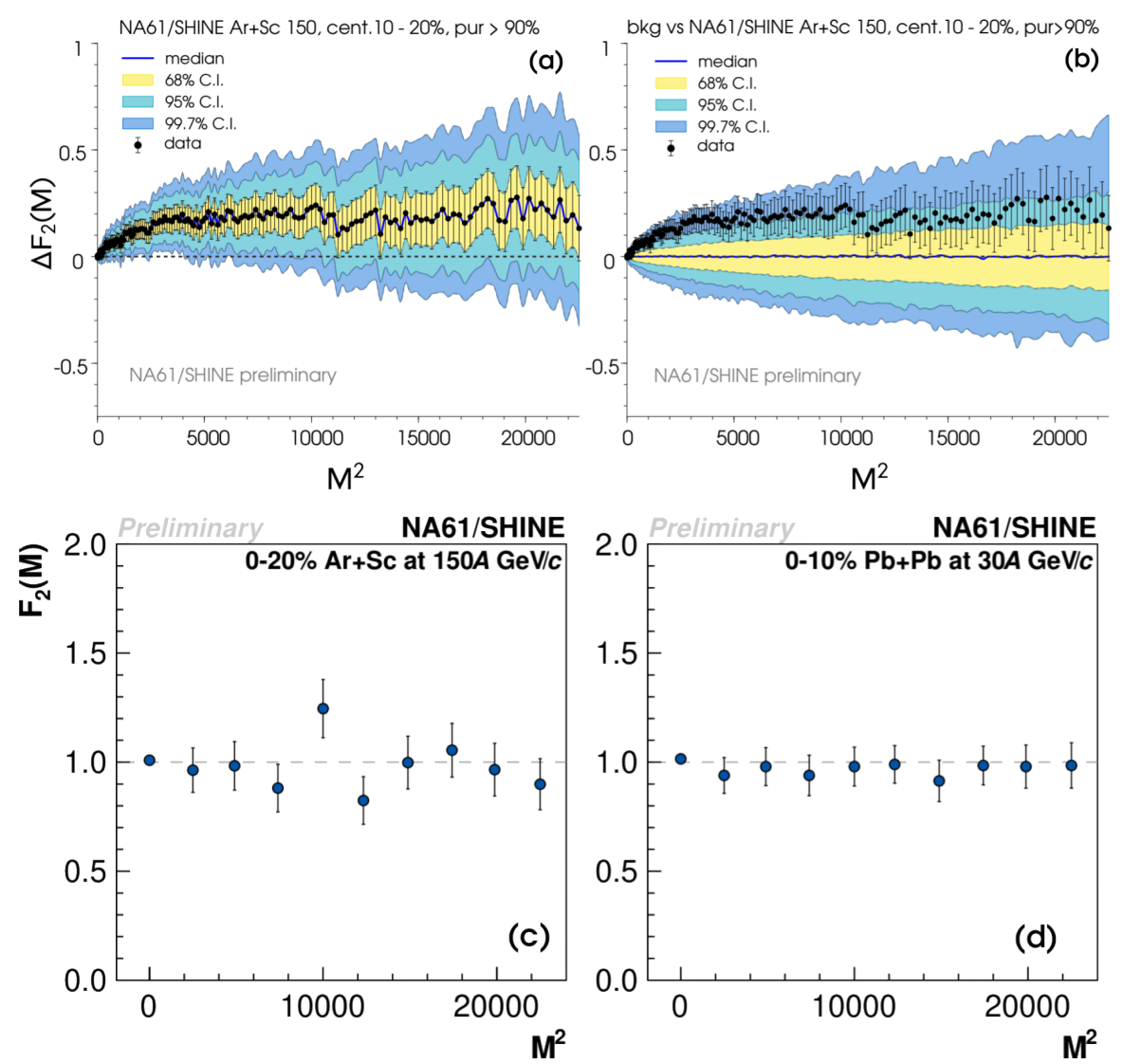

Figure 3: (Top row) (a) Correlated bin analysis results for $\Delta F_{2}(M)$ of NA61/SHINE Ar+Sc 10-20\% most central collisions @ 150A GeV/c [12], compared to $(b)$ random proton Monte Carlo background $\Delta F_{2}(M)$. (Bottom row) (c) Independent bin analysis [11] results for $F_{2}(M)$ of NA61/SHINE Ar+Sc 0-20\% most central collisions @ 150A GeV/c \& (d) Pb+Pb 0-10\% most central collisions @ 30A GeV/c [T. Czopowicz, C.P.O.D. 2021 Online]

[5] N.G. Antoniou, Y.F. Contoyiannis, F.K. Diakonos, A.I. Karanikas and C.N. Ktorides, Pion production from a critical QCD phase, Nucl. Phys. A 693 (2001) 799 [hep-ph/0012164].

[6] N.G. Antoniou, Y.F. Contoyiannis, F.K. Diakonos and G. Mavromanolakis, Critical QCD in nuclear collisions, Nucl. Phys. A 761 (2005) 149 [hep-ph/0505185].

[7] N.G. Antoniou, F.K. Diakonos, A.S. Kapoyannis and K.S. Kousouris, Critical opalescence in baryonic QCD matter, Phys. Rev. Lett. 97 (2006) 032002 [hep-ph/0602051].

[8] A. Bialas and R.C. Hwa, Intermittency parameters as a possible signal for quark - gluon plasma formation, Phys. Lett. B 253 (1991) 436.

[9] W.J. Metzger, "Estimating the uncertainties of factorial moments." HEN-455 (unpublished), (2004).

[10] N.G. Antoniou et al., Decoding the QCD critical behaviour in A + A collisions, Nucl. Phys. A 1003 (2020) 122018 [2004 . 12133].

[11] A. Bialas and M. Gazdzicki, A new variable to study intermittency, Physics Letters B 252 (1990) 483.

[12] N. Davis [NA61/SHINE collaboration], Searching for the Critical Point of Strongly Interacting Matter in Nucleus-Nucleus Collisions at CERN SPS, Acta Phys. Polon. Supp. 13 (2020) 637 [2002 . 06636]. 